\title{
Control of magnetic anisotropy by orbital hybridization with charge transfer in $\left(\mathrm{La}_{0.67} \mathrm{Sr}_{0.33} \mathrm{MnO}_{3}\right)_{n} /\left(\mathrm{SrTiO}_{3}\right)_{n}$ superlattice
}

Bangmin Zhang ${ }^{1}$, Lijun Wu $\mathbb{1}^{2}$, Jincheng Zheng $\mathbb{\circledR}^{2,3,4}$, Ping Yang ${ }^{5}$, Xiaojiang Y Y ${ }^{5}$, Jun Ding ${ }^{1}$, Steve M. Heald $\mathbb{0}^{6}$, Richard A Rosenberg ${ }^{6}$, Thirumalai Venky Venkatesan ${ }^{1,7,8,9,10}$, Jingsheng Chen (1) ', Cheng-Jun Sun ${ }^{6}$,

Yimei Zhu ${ }^{2}$ and Gan Moog Chow ${ }^{1}$

\begin{abstract}
The chemical asymmetry at the hetero-structure interface offers an effective opportunity to design desirable electronic structures by controlling charge transfer and orbital hybridization across the interface. However, controlling the hetero-interface remains a daunting task. Here, we report the modulation of interfacial coupling of $\left(\mathrm{La}_{0.67} \mathrm{Sr}_{0.33} \mathrm{MnO}_{3}\right)_{n} /$ $\left(\mathrm{SrTiO}_{3}\right)_{n}$ superlattices by manipulating the periodic thickness with $n$ unit cells of $\mathrm{SrTiO}_{3}$ and $n$ unit cells of $\mathrm{La}_{0.67} \mathrm{Sr}_{0.33} \mathrm{MnO}_{3}$ with a fixed thickness of $\sim 120$ unit cells. The easy axis of magnetic anisotropy rotates $\sim 45^{\circ}$ towards the out-of-plane direction from $n=10$ to $n=2$ at reduced temperature $T_{\mathrm{Re}}=T / T_{\mathrm{S}}=0.87$, where $T_{\mathrm{S}}$ is the temperature at the onset of magnetization. Transmission electron microscopy reveals an enlarged tetragonal ratio $>1$ with breaking of volume conservation around the $\left(\mathrm{La}_{0.67} \mathrm{Sr}_{0.33} \mathrm{MnO}_{3}\right)_{n} /\left(\mathrm{SrTiO}_{3}\right)_{n}$ interface and electronic charge transfer from $\mathrm{Mn}$ to $\mathrm{Ti}$ $3 d$ orbitals across the interface. Orbital hybridization accompanying the charge transfer results in preferred occupancy of $3 d_{3 z^{2}-r^{2}}$ orbitals at the interface and induces a stronger electronic hopping integral and interfacial magnetic anisotropy along the out-of-plane direction, which contributes to the rotation towards the out-of-plane direction of an effective magnetic easy axis for $n=2$. We demonstrate that interfacial orbital hybridization with charge transfer in the superlattice of strongly correlated oxides may be a promising approach to tailor electronic and magnetic properties in device applications.
\end{abstract}

\section{Introduction}

The asymmetry at the heterostructure interface of $3 d$ transitional metal $\mathrm{ABO}_{3}$ oxides, including the mismatch of lattice constant, oxygen octahedral rotation and distortion, and chemical environment, has profound influences on spin and orbital coupling, yielding emerging phenomena such as enhanced ordering temperature, induced interfacial magnetism at the superconductor/

\footnotetext{
Correspondence: Cheng-Jun Sun (cjsun@aps.anl.gov) or Yimei Zhu

(zhu@bnl.gov) or Gan Moog Chow (msecgm@nus.edu.sg)

${ }^{1}$ Department of Materials Science \& Engineering, National University of

Singapore, 9 Engineering Drive 1, Singapore 117576, Singapore

${ }^{2}$ Condensed Matter Physics \& Materials Science Division, Brookhaven National

Laboratory, Upton, NY 11973, USA

Full list of author information is available at the end of the article.

These authors equally contributed to the work: Bangmin Zhang, Lijun Wu
}

manganite interface and orbital reconstruction ${ }^{1-11}$. Coupling between crystal structures ${ }^{12-18}$ across the heterostructure interface leads to new properties, a filmthickness-dependent interfacial ferromagnetic-polaronic insulator in $\mathrm{Pr}_{0.67} \mathrm{Sr}_{0.33} \mathrm{O}_{3} / \mathrm{SrTiO}_{3}{ }^{19}$, spontaneous deep polarization ${ }^{15}$ in $\mathrm{SrTiO}_{3}$ near the $\mathrm{La}_{2 / 3} \mathrm{Sr}_{1 / 3} \mathrm{MnO}_{3} / \mathrm{SrTiO}_{3}$ interface, rotation of the magnetic easy axis of $\mathrm{La}_{2 / 3} \mathrm{Sr}_{1 /}$ ${ }_{3} \mathrm{MnO}_{3}{ }^{20}$ and $\mathrm{SrRuO}_{3}{ }^{21}$ thin film. Most work focuses mainly on the crystal structure without explicitly considering the effect of charge transfer. In $3 d$ oxides, the properties of transition metal oxides are sensitive to electronic structure, especially the occupation of $3 d$ orbitals ${ }^{4}$, which may be manipulated by doping with different covalent ions ${ }^{22}$, hydraulic pressure ${ }^{23}$, and external electric bias ${ }^{24,25}$. The chemical asymmetry, such as polar 
discontinuity, of the $\mathrm{ABO}_{3}$ perovskite heterostructure interface may be exploited to study the effect of charge transfer and crystal structure ${ }^{26-28}$.

Recent work ${ }^{13}$ claimed that oxygen octahedral coupling (OOC) is the leading factor affecting orbital hybridization in ultrathin manganite film; however, the effect of charge transfer on magnetic properties is also important ${ }^{6-11}$. Here, we consider a model system $-\mathrm{La}_{0.67} \mathrm{Sr}_{0.33} \mathrm{MnO}_{3} /$ $\mathrm{SrTiO}_{3}$ (LSMO/STO) superlattice deposited on an (001) STO substrate. Bulk LSMO with high Curie temperature and high spin polarization ${ }^{28}$ has a rhombohedral unit cell $^{29}$ with space group $R-3 c$ (No. 167) and lattice constants $a_{r}=5.471 \AA$ and $\alpha_{r}=60.43^{\circ}$ at room temperature. The calculated pseudocubic lattice constant is $3.880 \AA$, and the $\mathrm{MnO}_{6}$ rotation pattern is $a^{-} a^{-} a^{-}$with rotation angle $2.2^{\circ}$. STO is cubic ${ }^{30}$ at room temperature with lattice constant $\sim 3.905 \AA$ close to the lattice constant of LSMO, without $\mathrm{TiO}_{6}$ rotation. For the (001)-oriented heterostructure, the $\mathrm{TiO}_{2}$ and $\mathrm{SrO}$ atomic planes of STO are charge-neutral (no planar charge), while $\mathrm{MnO}_{2}$ and $\mathrm{La} / \mathrm{SrO}$ atomic planes of LSMO have negative and positive planar charge, respectively, as illustrated as a blue bar at right bottom of Fig. 1a. The polar discontinuity exists around the LSMO/STO heterostructure, and the charge transfer across the interface occurs to avoid the polar catastrophe $^{31,32}$. The final distribution of planar charge is illustrated as a pink bar at the right bottom of Fig. 1a, and the short-range charge transfer is constrained to several unit cells (UCs) near the interface ${ }^{32-35}$, illustrated as the probability of charge transfer at the left bottom of Fig. 1a. The superlattice with multiple heterostructure interfaces provides a viable way to manipulate the intensity of interfacial coupling, which may facilitate the short-range charge transfer.

In this work, the interfacial coupling of $(\mathrm{LSMO})_{n} /(\mathrm{STO})_{n}$ superlattices (SLs) is manipulated by controlling the periodic thickness with $n$ UCs of STO and $n$ UCs of LSMO with a fixed thickness of $\sim 120$ UCs. The easy axis of magnetic anisotropy rotates $\sim 45^{\circ}$ towards the out-of-plane direction from $n=10$ to $n=2$ at reduced temperature $T_{\operatorname{Re}}=T / T_{\mathrm{S}}=0.87$, where $T_{\mathrm{S}}$ is the onset point of magnetization. Orbital hybridization accompanying charge transfer from Mn $3 d$ and Ti $3 d$ orbitals results in preferred occupancy of the $3 d_{3 z^{2}-r^{2}}$ orbital at the interface and induces a stronger electronic hopping integral and interfacial magnetic anisotropy along the out-of-plane direction. As a result, the rotation to the out-of-plane direction of the effective magnetic easy axis for $n=2$ is observed. This work reveals the predominant effect of charge transfer on the orbital hybridization and interfacial magnetic anisotropy.

\section{Materials and methods}

$\left[(\mathrm{LSMO})_{n} /(\mathrm{STO})_{n}\right]_{m}$ SLs with different periodic thickness ( $n$ UC LSMO and $n$ UC STO, SL $n$ ), as illustrated in
Fig. 1a, were grown on the (001) STO substrate by pulsed laser deposition at 100 mTorr oxygen pressure with the substrate temperature at $780^{\circ} \mathrm{C}$. Before film deposition, the $\mathrm{SrTiO}_{3}$ substrate was treated with a buffered $\mathrm{NH}_{4} \mathrm{~F}$ HF solution, then annealed at $975^{\circ} \mathrm{C}$ for $2 \mathrm{~h}$ to obtain $\mathrm{TiO}_{2}$ plane termination. The reflection high-energy electron diffraction (RHEED) was used to monitor the deposition rate. The energy density of the $248 \mathrm{~nm} \mathrm{KrF}$ excimer laser beam on target was $1.5 \mathrm{~J} / \mathrm{cm}^{2}$ at a pulse frequency of $2 \mathrm{~Hz}$. After deposition, the sample was cooled at $15^{\circ} \mathrm{C} / \mathrm{min}$ in a 200 -mTorr oxygen atmosphere. The top surface is $n$ UC STO, and the total thickness was kept at $2 n \times m=120 \mathrm{UC}$ (except for $n=8$, for which the total thickness is $128 \mathrm{UCs}$ ). The final surface morphology with roughness $\sim 0.3 \mathrm{~nm}$ was measured by atomic force microscopy (Fig. S1). The crystallographic properties of the films at room temperature were studied using a fourcircle diffractometer (Huber 4-circle system 90000-0216/ 0 ) at the Singapore Synchrotron Light Source (SSLS), with an X-ray wavelength equivalent to $\mathrm{Cu} K_{\alpha 1}$ radiation. The magnetic properties were measured by a superconducting quantum interference device (SQUID), and the diamagnetic signal from the STO substrate was subtracted to obtain the magnetic signal from LSMO/STO superlattice only. The magnetization value is calculated by $M /\left(\frac{A}{a^{2}} t\right)$, where $M$ is the measured magnetic moment, $A$ is the inplane area of the sample, $a$ is the in-plane lattice constant (which is the same as the in-plane lattice constant of the STO substrate), and $t$ is the thickness of the LSMO of the whole sample in number of UCs (64 for SL8, and 60 for all other samples). The transport properties were measured by the Physical Property Measurement System (PPMS) using a linear four-point probe. The X-ray absorption near edge structure (XANES) measurements were carried out using linear polarized X-rays at the undulator beamline 20-ID-B of the Advanced Photon Source (APS), Argonne National Laboratory (ANL), USA. The Si (111) monochromator with resolution $\delta E / E=1.3 \times 10^{-4}$ was used. The XANES was collected in the fluorescence mode using a 12-element Ge solid-state detector. A Mn metal foil, placed to intercept a scattered beam, was used as an online check of the monochromator energy calibration. To get reliable information, each XANES curve was obtained by averaging the results of five measurements with total counts of more than one million. The X-ray magnetic circular dichroism (XMCD) with different incident angles at $L_{3,2}$ edges of Mn was measured with a magnetic field of $5 \mathrm{kOe}$ parallel to the wave vector of the $\mathrm{X}$-ray, at 4-ID-C, APS in ANL and SINS beamline in SSLS, using the total electron mode for data collection at different temperatures. After the raw absorption spectra were normalized to the incoming photon intensity, a linear background obtained by fitting the absorption at the pre-edge range was subtracted, and the absorption at 


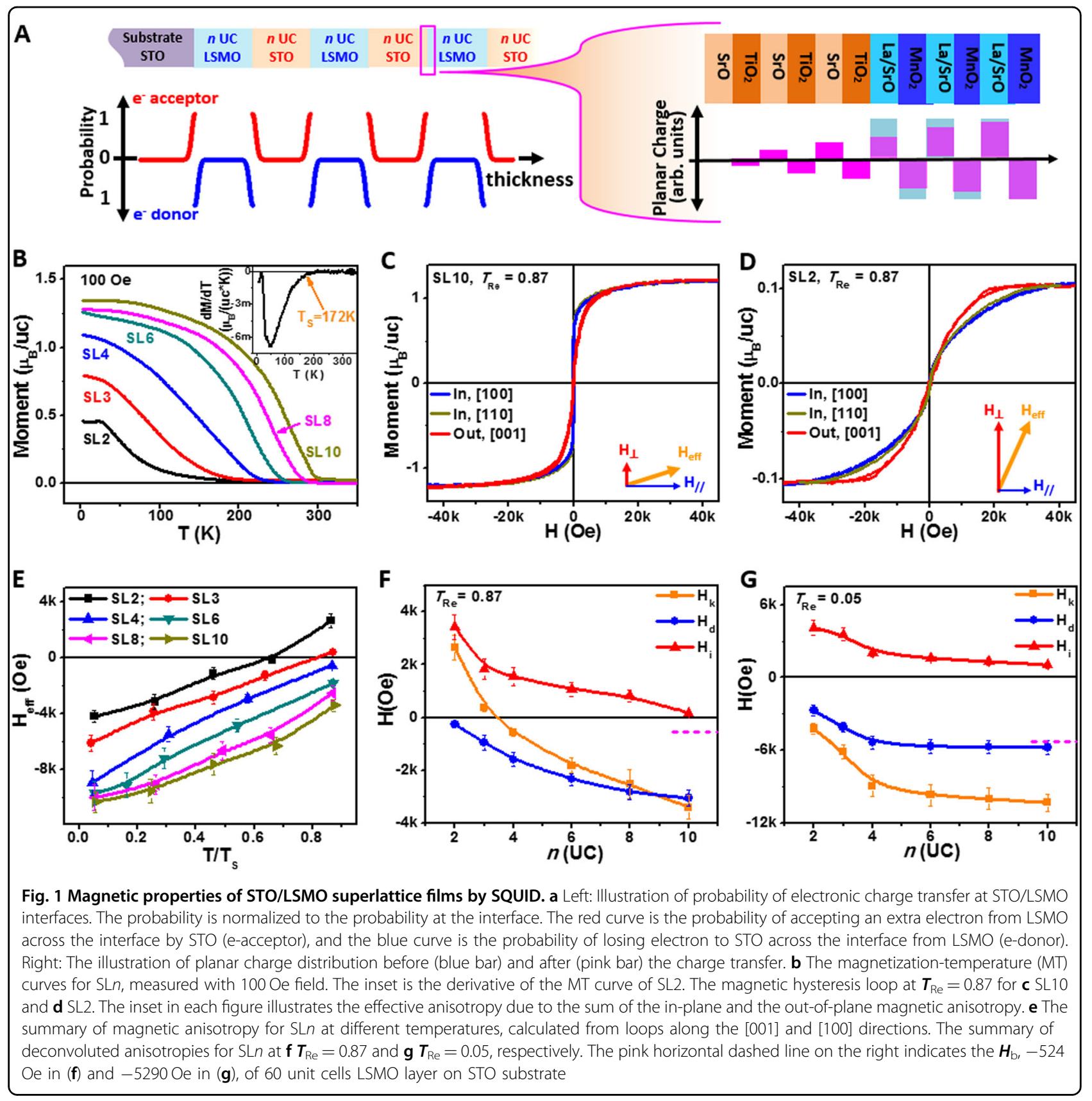

the pre-edge was at zero intensity. The absorption spectra were normalized to the post-edge of absorption. The normalized XMCD ${ }^{36}$ was obtained by $\left(\rho^{+}-\rho^{-}\right) /\left(\rho^{+}+\rho^{-}\right)$, where $\rho^{+}$and $\rho^{-}$are absorption with the helicity parallel and antiparallel to the applied magnetic field, respectively. The first-principle simulation of X-ray absorption spectroscopy (XAS) and XMCD was conducted using the FDMNES code ${ }^{37,38}$, developed by Yves Joly, Yvonne Soldo-Olivier et al. at Institut Néel, France. The superlattice structure was built based on experimental results. The final states of the electrons in the cluster with radius of $6 \AA$ were calculated using the multiple-scattering approach with spin-orbit coupling included, and the chemical valence of $\mathrm{Mn}$ was controlled by setting the number of $3 d$ electrons accordingly. To account for the core-hole lifetime, the calculated XAS and XMCD spectra at $\mathrm{Mn} L$ edges were convoluted with a Lorentzian function. In addition, to account for the experimental resolution and scaling of the spectra, a complementary convolution with a Gaussian function with typical broadening parameters of $\sigma=1.0 \mathrm{eV}$ was performed. The spectra of $\mathrm{Mn}$ with various chemical valences are 
normalized for easy comparison. Select area electron diffraction (SAED), high resolution TEM (HRTEM), scanning transmission electron microscopy-high angle annular dark field (STEM-HAADF), and electron energy loss spectroscopy (EELS) spectrum imaging were performed using the double aberration-corrected JEOLARM200CF microscope with a cold-field emission gun and operated at $200 \mathrm{kV}$, at Brookhaven National Laboratory, USA. The microscope is equipped with JEOL and Gatan HAADF detectors for incoherent HAADF (Zcontrast) imaging, Gatan GIF Quantum ER Energy Filter with dual EELS for EELS. The cross-section TEM samples were prepared by focused ion beam (FIB) followed by gentle ion-milling at low operating voltages. The STEMHAADF images were acquired with a condensed aperture of $21.2 \mathrm{mrad}$ and collection angle of $67-275 \mathrm{mrad}$.

\section{Results and discussion}

The Magnetization-Temperature (MT) curves in Fig. 1b show that the paramagnetic-to-ferromagnetic phase transition temperature decreases with decreasing $n$. In the double-exchange model ${ }^{22}$, ferromagnetic exchange between $\mathrm{Mn}^{3+}$ and $\mathrm{Mn}^{4+}$ ions occurs via the oxygen $2 p$ orbital. An electron from $\mathrm{Mn}^{3+}$ transfers to the oxygen simultaneously with oxygen transferring an electron to $\mathrm{Mn}^{4+}$. This transfer effectively facilitates the movement of spin from a $\mathrm{Mn}^{3+}$ ion to a $\mathrm{Mn}^{4+}$ ion without spin flip. The delocalization of the electron reduces the kinetic energy, thus enabling the ferromagnetic exchange. The intensity of this double-exchange interaction depends on the local atomic and chemical environment. At the LSMO/STO interfaces, the magnetic exchange interaction is weakened, which is observed when the $n$ decreases (the number of LSMO/STO interfaces increases) the temperature of phase transition of the LSMO decreases. The inset of Fig. 1b shows the broad magnetization transition for SL2, which does not allow for an unambiguous definition of the Curie temperature. Then, a different reference temperature $T_{\mathrm{S}}$ is introduced for analysis, defined as the temperature at which magnetization of the material starts to appear. The derivative of MT curve shows that the magnetic moment starts to increase at $T_{\mathrm{S}}=172 \mathrm{~K}$ in SL2. At the beginning of the phase transition, a cluster or spin glass state may exist due to the spin frustration at LSMO/STO interface, to be discussed later. To compare magnetic anisotropy between different samples, the reduced temperature $T_{\mathrm{Re}}=T / T_{\mathrm{S}}$ is employed. The summary of $T_{\mathrm{S}}$ and $T_{\mathrm{Re}}$ is shown in Table S1 for different samples. At $T_{\mathrm{Re}}=0.87$, Fig. 1c, d shows that the out-ofplane [001] axis of SL2 is easier to saturate than the in-plane [100] axis, which is opposite for SL10. These hysteresis loops ${ }^{30}$ clearly show a change in magnetic easy axis direction between SL2 and SL10. See Fig. S2 for more hysteresis loops. The saturated magnetization of bulk
$\mathrm{LSMO}^{22}$ is $3.7 \mu_{\mathrm{B}} / \mathrm{uc}$, higher than the saturated magnetization of the film in the current work. The weakening of magnetic properties of manganite thin films has previously been observed ${ }^{39}$. The decrease in the saturated magnetization with $n$ suggest that the effect of the LSMO/ STO interfaces on the magnetic properties is significant. The hysteresis loop along the [110] is quite close to that along the [100] direction in Fig. 1c, d, and there is a weak in-plane anisotropy with the easy axis along the [110] direction. Similar in-plane weak anisotropy has been reported in a single LSMO layer on the [001] STO substrate $^{39}$. From the area enclosed by the in-plane and the out-of-plane hysteresis loops (effective magnetic anisotropy energy, $K_{\text {eff }}$ ) in the first quadrant, the effective magnetic anisotropy could be calculated via $H_{\text {eff }}=-2 K_{\text {eff }} /$ $\mu_{0} M_{\mathrm{S}}$, where $H_{\text {eff }}$ is the effective magnetic anisotropy field, and $M_{\mathrm{S}}$ is the saturated magnetization. For SL2, the difficulty of saturating the material along different directions follows the sequence: $[001]<<[110]<[100]$. Although for SL10 the sequence of difficulty to saturate the material is $[110]<[100]<<[001]$, the energy difference required to saturate the sample between [110] and [100] directions is quite small, as shown in Fig. 1c. Then, magnetic anisotropy between [001] and [100] directions is discussed, unless specified. The small remnant of magnetization in SL2 depends on the magnetic domain structure and the magnetization process ${ }^{40}$, which is not in the scope of this work.

Figure 1e summarizes $H_{\text {eff }}$ for all SLs at different temperatures. The positive value of $H$ denotes an easy axis normal to the film plane, and the negative value denotes an easy axis in the film plane. With increasing temperature and decreasing $n$, the magnetic anisotropy favoring in-plane decreases gradually (negative $H_{\text {eff }}$ approaching zero) for all samples, and SL2 shows a magnetic easy axis favoring the out-of-plane direction at high temperature $T_{\mathrm{Re}}=0.87$ with positive $H_{\text {eff. }} H_{\text {eff }}$ could be viewed as the sum of terms favoring the in-plane orientation $\left(H_{/ /}\right)$and that favoring the out-of-plane orientation $\left(H_{\perp}\right)$, as illustrated in the inset of Fig. 1c, d. The effective anisotropy field $^{41}$ is deconvoluted as $H_{\text {eff }}=H_{\mathrm{i}}+H_{\mathrm{b}}-H_{\mathrm{d}}$, including bulk term $H_{\mathrm{b}}$ and demagnetization term $H_{\mathrm{d}}$ favoring the in-plane direction, and interfacial term $H_{\mathrm{i}}$ favoring the out-of-plane direction. The demagnetization term could be calculated by $H_{\mathrm{d}}=-\mathrm{N} \cdot \mathrm{M}_{\mathrm{S}}$, while the demagnetization factor is $N=1$ along the out-of-plane direction for thin films. $H_{\mathrm{b}}+H_{\mathrm{i}}$ is the difference between $H_{\text {eff }}$ and $H_{\mathrm{d}}$. For different samples, all the growth parameters such as temperature, oxygen pressure, and laser energy are the same, and the only difference is the number of LSMO/ STO interfaces. Any change in $H_{\mathrm{b}}+H_{\mathrm{i}}$ with $n$ should therefore come from the LSMO/STO interfacial coupling, and the bulk term $H_{\mathrm{b}}$ should be constant for different samples. In this work, the $H_{\mathrm{b}}$ is obtained from a reference 
sample of 60 UC single layer LSMO on the (001) STO substrate (without depositing any STO layers), the interfacial term $H_{\mathrm{i}}$ of which is ignored. Due to the multiple contributions to $H_{\text {eff }}$, this parameter experiences a change in direction of magnetic easy axis with $n$ and temperature. Figure 1f shows the trend of deconvoluted anisotropies with $n$ at $T_{\mathrm{Re}}=0.87$ and the constant bulk $H_{\mathrm{b}}$ term (dashed horizontal pink line). The demagnetization effect $H_{\mathrm{d}}$ decreases with decreasing $n$ due to decreasing $M_{\mathrm{S}}$. For $n=2, H_{\mathrm{d}}$ is close to zero due to the low magnetization $M_{\mathrm{S}}=0.11 \mu_{\mathrm{B}} /$ uc. $H_{\mathrm{i}}$ increases with decreasing $n$, which, complementary to the decreased $H_{\mathrm{d}}$, induces rotation of the easy axis towards the out-of-plane direction in SL2. Current experimental results reveal that multiple interfaces in SLs render $H_{\mathrm{i}}$ non-trivial.

With decreasing temperature, the magnetic anisotropy favors the in-plane direction for all samples. Taking $T_{\mathrm{Re}}$ $=0.05$ as an example, Fig. 1g shows the trend of deconvoluted anisotropies with $n . H_{\mathrm{i}}$ increases with decreasing $n$, following the similar trend at high temperatures (Fig. 1f). Compared to high temperature $T_{\mathrm{Re}}=0.87$, at low temperatures $T_{\mathrm{Re}}=0.05 H_{\mathrm{i}}$ increases slightly. However, the bulk term $H_{\mathrm{b}}$ and the demagnetization term $H_{\mathrm{d}}$ are significantly enhanced. As a result, the effective anisotropy $H_{\text {eff }}$ favors the in-plane direction at $T_{\mathrm{Re}}=0.05$. In addition to rotating the direction of the easy axis, the interfacial coupling could induce a certain degree of spin frustration around the interface ${ }^{42}$, as revealed by the magnetic memory effect as shown in Fig. S3. The memory effect is obvious in SL2 at $15 \mathrm{~K}$ but is relatively weak in SL6 and SL10. These results suggest that the spin frustration may be related to the LSMO/STO interfacial coupling, i.e., a higher number of LSMO/STO interfaces promotes stronger spin frustration. The enhanced spin frustration in SL2 may contribute to the broad magnetization transition as shown in Fig. 1b. The above discussion reveals that the interracial coupling exists in a wide temperature range.

Angularly dependent $\mathrm{Mn} L_{3,2}$ edge XMCD yields element-specific information of magnetic anisotropy at different temperatures, as shown in Fig. 2 and Fig. S4. The incidence angle $\theta=0^{\circ}$ corresponds to the wave vector of the X-ray along the normal direction of the film plane, and the magnetic field is always parallel to the wave vector. The probing depth of XMCD is governed by the electron escape depth $\left(\lambda_{e}\right.$, several $\left.\mathrm{nm}\right)$, independent of the incident angles for the same sample. A simplified estimation of the saturation effect ${ }^{43}$ of angularly dependent $\mathrm{XMCD}$ has been corrected by a factor $f=\frac{1}{1+\lambda_{e} /\left(\lambda_{x} \cos \theta\right)} . \lambda_{e}$ is in the range of several $\mathrm{nm}^{44}$, and $\lambda_{e}=5 \mathrm{~nm}^{45}$ is an
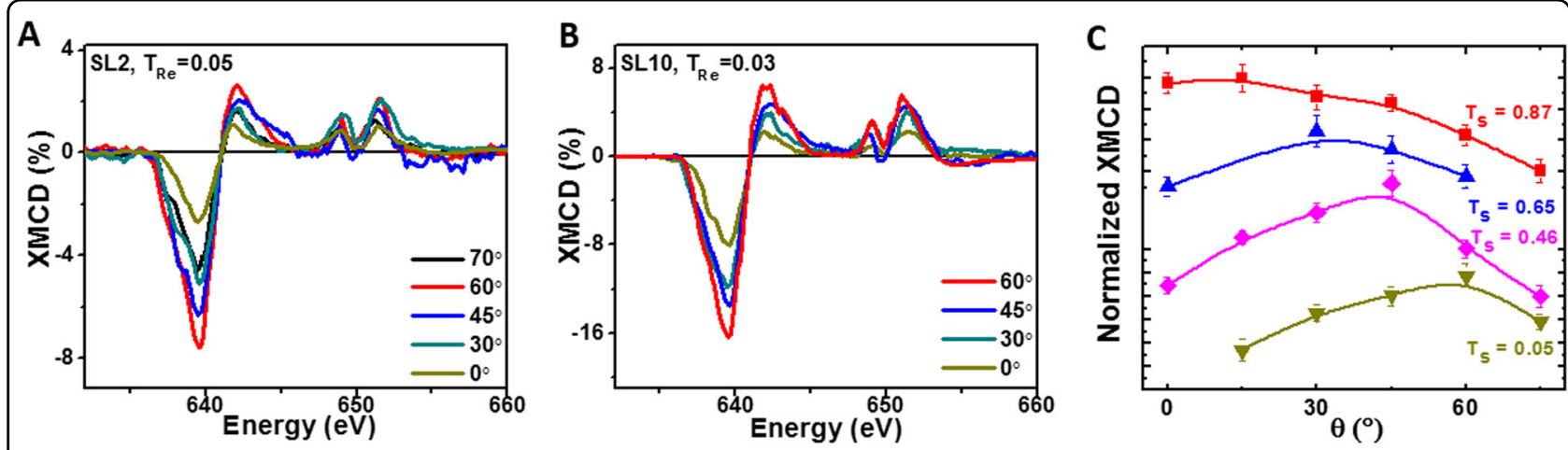

D

E
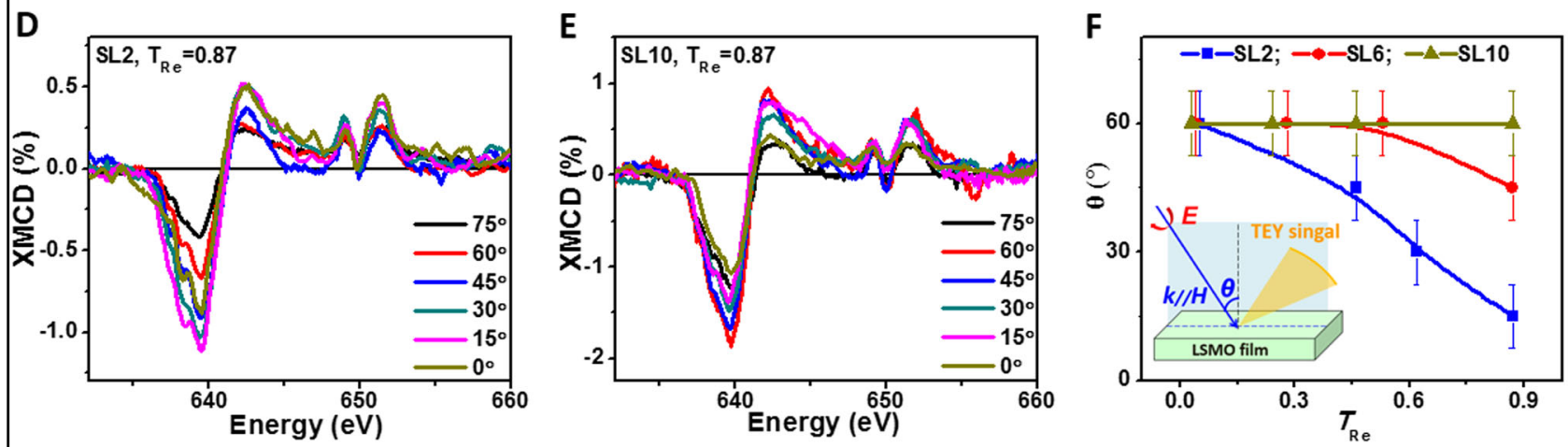

Fig. 2 Angular Mn L edge XMCD for SL2, SL6 and SL10 STO/LSMO superlattice films. Angular dependent Mn $L_{3,2}$ edge XMCD for SL2 (a, b), and SL10 (d, e) at different temperatures. The XMCD intensity at $639 \mathrm{eV}$ is summarized in (c) to determine the easy axis of the SL2 at different temperatures. The curves for different temperatures are vertically shifted for comparison. $\mathbf{f}$ The summary of the incident angle $\boldsymbol{\theta}$ with maximum XMCD intensity for SL2, SL6, and SL10 at different temperatures. Inset of $(\mathbf{f})$ is the setup for the measurement 
appropriate estimation for calculation in manganite; $\lambda_{x} \cos \theta$ is the $\mathrm{X}$-ray penetration depth. $\lambda_{x}$, X-ray penetration length, around the $\mathrm{Mn} L$ absorption edge is estimated to be $\sim 198 \mathrm{~nm}$ according to the previous report ${ }^{46}$, ignoring the energy dependence of $\lambda_{x}$ as discussed in ref. ${ }^{47}$. Then, the magnetic easy axis of a particular sample is identified by the maximum XMCD intensity at different incident angles. Figure $2 \mathrm{c}$ shows the summary of XMCD intensity at the $L_{3}$ edge, $\sim 639 \mathrm{eV}$ for SL2. With decreasing temperature $T_{\text {re }}$ from 0.87 to $\sim 0.05$, the easy axis rotates from $15^{\circ}$ to $60^{\circ}$ for SL2. However, the easy axis does not change for SL10 $\left(60^{\circ}\right)$ in the temperature range shown in Fig. 2 f. At the same reduced temperature $T_{\mathrm{re}}=0.87$, the direction with maximum XMCD intensity is $\theta=60^{\circ}, 45^{\circ}$, and $15^{\circ}$ for $n=10,6,2$, respectively. In SL10, the LSMO/ STO interfacial coupling still exists, including the coupling of lattice constant and charge transfer as discussed later. The interfacial contribution $H_{\mathrm{i}}$ may cause the easy axis at $60^{\circ}$ with respect to the surface normal rather than being completely in the in-plane direction. Compared to SL10, the easy axis of magnetic Mn ions in SL2 rotates $45^{\circ}$ towards the out-of-plane direction, which is consistent with the SQUID results. The maximum intensity at $60^{\circ}$ for SL10 at low temperature $T_{\text {re }}=0.03$ in Fig. $2 \mathrm{~b}$ shows the same trend of establishment of the magnetic easy axis towards the in-plane direction. Angular dependent XMCD therefore indicates that Mn ions have an important contribution to the rotation of magnetic anisotropy in SLn. The contribution from the Ti sites to the total magnetic moment is insignificant as calculated by the sum rules $^{48}$ from Ti $L_{3,2}$ XMCD (Fig. S5). Hence, the discussion of magnetic properties focuses on Mn sites hereafter.

Both the SQUID and angularly dependent XMCD reveal an out-of-plane magnetic easy axis for SL2 at $T_{\mathrm{re}}=$ 0.87 due to the strong interfacial contribution. The effect of interfacial OOC on magnetic anisotropy has been investigated recently ${ }^{13,20,21,49}$. In this work, quantitative analysis of the crystal structure using X-ray diffraction, half-integer diffraction ${ }^{50-52}$ (Fig. S6, and Table S2), detecting three-dimensional $\mathrm{MnO}_{6}$ rotation, shows a large difference of the $\mathrm{Mn}-\mathrm{O}$ bond angle and bond length along the in-plane and the out-of-plane directions in SL10. In SL2, although the measured bond lengths of both directions are similar, the larger in-plane bond angle (Fig. S6B) suggests a higher in-plane hopping integral, and the magnetic moment would favor the [100] axis instead of the [001] axis, as observed. ref. ${ }^{13}$ claimed that OOC is the leading factor affecting orbital hybridization to determine material properties. However, octahedral rotation cannot explain the results of the current LSMO/ STO superlattice. Other possible factors include the strong $3 d$ spin $-5 d$ orbital coupling ${ }^{53}$, as proposed in the $\mathrm{La}_{2 / 3} \mathrm{Sr}_{1 / 3} \mathrm{MnO}_{3} / \mathrm{SrIrO}_{3}$ interface, which is relatively weak between the $3 d$ transition metals $(\mathrm{Mn}$ and $\mathrm{Ti}$ ) in the LSMO/STO superlattice. Therefore, it is necessary to consider other factors responsible for the observed phenomena. Then, high resolution scanning transmission electron microscopy (STEM) was used to probe the local structural information at the LSMO/STO interfaces for $n=2,6,10$ in Fig. 3. The STO substrate, at the left side of the image, was treated with a $\mathrm{TiO}_{2}$ termination before deposition, and atomically flat LSMO/STO interfaces existed in these samples.

By refining the peak positions, the (001) plane spacing of two successive $\mathrm{La} / \mathrm{SrO}$ (or $\mathrm{SrO}$ ) planes along the [001] direction $\left(c_{\text {int }}\right)$ was obtained. For SL10 and SL6, the $c_{\text {int }}$ shows an oscillating pattern with a maximum value at the left STO/LSMO interface of each LSMO layer, larger than $3.905 \AA$, and with a minimum at the right LSMO/STO interface of each LSMO layer, which indicates an asymmetric coupling at two interfaces of each LSMO layer and is related to the growth process (to be discussed later). The oscillation pattern of $c_{\text {int }}$ in film is further confirmed by geometric phase analysis (GPA) $)^{54,55}$ from the HRTEM image (the second row of Fig. 3e). There is basically no change in the in-plane lattice (the third row in Fig. 3e) over the whole image except some random changes caused by the image noise. For SL2, there is no obvious pattern of $c_{\text {int }}$, but $c_{\text {int }}$ of the whole film is slightly larger $\left(c_{\text {int }} / a>1\right)$ than $c_{\text {int }}$ of the substrate STO, revealed as the dominant red part of GPA (second raw of Fig. 3f). The SAED is used to separate the contribution of the diffraction peak between the film and the substrate in Fig. S7. The 002 diffraction peak of SL2 film (blue line) clearly shifts to the left with respect to the substrate (red line), confirming that the $c_{\text {int }}$ of the SL2 film is slightly larger than the $c_{\text {int }}$ of the STO substrate. The SAED reveals that $a=3.905 \AA$, and $c_{\text {int }}=3.911 \AA$ for SL2, which is consistent with the XRD results in Fig. S8. The shoulder at the right side of the $002 \mathrm{X}$-ray diffraction reflection, corresponding to the film, is quite clear for $n \geq 6$, but disappears for $n<4$, revealing that the out-of-plane lattice constant increases with the decrease in $n$ for SLn. A similar enlarged tetragonal ratio around the interface has been reported in other system $^{56}$, which may affect the electronic structure. In addition, the Mn displacement from the body center of the pseudocubic UC, recently reported by Guo et al. ${ }^{57}$, is observed and discussed in Fig. S9.

Volume conservation is generally not observed in epitaxial thin film ${ }^{58}$, due to the strain effect of the substrate or interfacial effects (such as chemical interdiffusion and charge transfer). In the case when the lattice constant is dominated by the substrate-induced strain effect ${ }^{58}$, the inplane tensile strain normally induces out-of-plane compressive strain with $c / a<1$. However, in our current work, the LSMO is under in-plane tensile strain with $a=3.905$ $\AA$, and the out-of-plane lattice around the LSMO/STO 

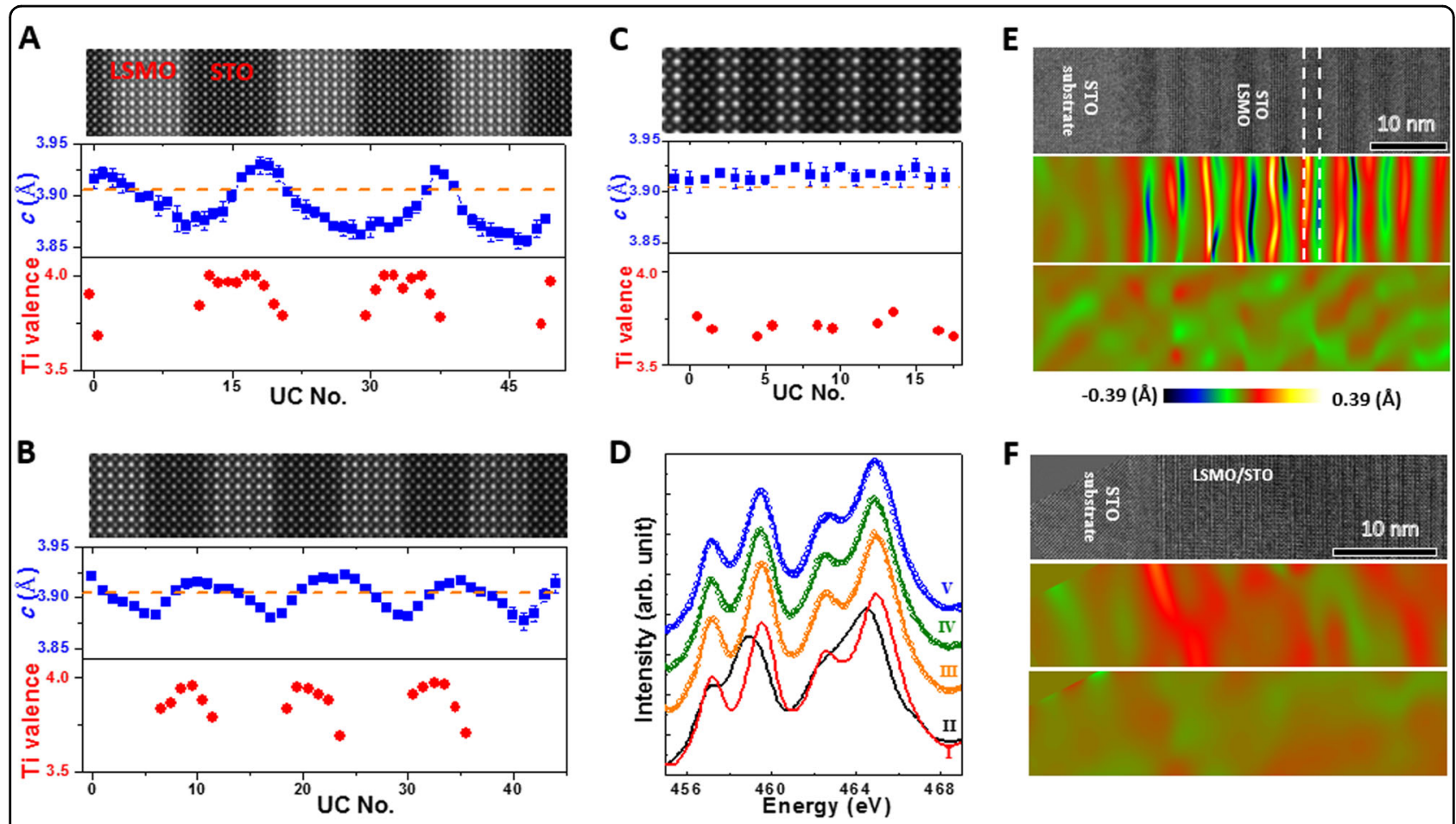

Fig. 3 HAADF-STEM images of (a-c) SL10, SL6, and SL2 STO/LSMO superlattice films. The out-of-plane plane spacing $c_{\text {int }}$ (blue squares) and Ti chemical valence (red dots) as a function of position are shown in the middle and bottom part of $\mathbf{a}-\mathbf{c}$. The horizontal orange dashed lines mark the $c_{\text {int }}$ of STO in the bulk substrate. d Ti EELS at $L_{3,2}$ edges from $\mathrm{SrTiO}_{3}$ substrate far from interface (I: red line), sample surface (II: black line), interface between LSMO/STO superlattice (III: orange circles), center of deposited STO layer in LSMO/STO superlattice (IV: olive circles) in SL6, and deposited STO layer in SL2 film (V: blue circles). The spectra of I and II are close to those of bulk $\mathrm{SrTiO}_{3}$ and $\mathrm{LaTiO}_{3}$, respectively and are thus used as the reference spectra of $\mathrm{Ti}^{4+}$ and $\mathrm{Ti}^{3+}$. The appearance of $\mathrm{Ti}^{3+}$ is due to the lack of oxygen coordination at the sample surface ${ }^{53}$. Then, the fitted $\mathrm{Ti}$ valence is $\mathrm{Ti}^{3.97+}, \mathrm{Ti}^{3.78+}$ and $\mathrm{Ti}^{3.75+}$ for III, $\mathrm{VI}$ and $\mathrm{V}$, respectively. e, $\mathbf{f}$ High resolution TEM (HRTEM) image for SL6 and SL2. The second and third rows correspond to the relative change in the out-of-plane (second row) and the in-plane (third row) spacing of SrO (La/SrO) planes. The vertical white dashed lines at two STO/LSMO interfaces are for eye guidance

interface is larger than the in-plane lattice constant $\left(c_{\text {int }}=\right.$ $3.911 \AA$ for SL2) with $c / a>1$. Hence, other factors should be present. Inspired by a high correlation between crystal and electronic structures in the $3 d$ oxide, the electronic charge distribution was examined by $\mathrm{Ti} L$ edge EELS $^{34,59,60}$, summarized in Fig. 3a-d. SL10 and SL6 show the same trend of Ti chemical valence. Taking SL6 as an example, the chemical valence of $\mathrm{Ti}$ at the LSMO/STO interface ( 3.78) decreases compared to the chemical valence of $\mathrm{Ti}$ at the center of the STO layer ( 3.97), indicating that extra electrons are accepted by the $\mathrm{Ti} 3 d$ orbitals at the LSMO/STO interface. The critical thickness of the charge transfer from the STEM results is $\sim 3$ UCs, consistent with the observations of others ${ }^{34}$. The averaged chemical valence of $\mathrm{Ti}$ is $\sim 3.75$ in SL2 with each STO UC situated at the LSMO/STO interface. There is no clear trend of peak position of $\mathrm{Mn}^{61,62}$ in Fig. S10, similar to ref. ${ }^{13}$. However, the shape of the absorption different for Mn with a different chemical valence, even though change in peak position may be too subtle to detect. The first principle XAS simulation was then carried out using
FDMNES $^{37,38}$, which is a method different from the configuration interaction model ${ }^{63,64}$ and could be used to investigate the effect of the heterostructure interface. The comparison of the shape of the X-ray absorption curve between the simulated and measured $\mathrm{Mn} L$ edge XAS in S10 suggests $M n$ chemical valence higher than the bulk value of 3.3. The EELS shows evidence of charge transfer from $\mathrm{Mn}$ to Ti $3 d$ orbitals across the LSMO/STO interface, and the probability is the highest for SL2 (lowest Ti valence) with the maximum number of interfaces. One other possible reason for the change in the $\mathrm{Ti}$ chemical valence is the oxygen stoichiometry around the LSMO/ STO interface. In this work, the LSMO and STO layers for all samples were fabricated using the same growth parameters, including the oxygen pressure. If the change in $\mathrm{Ti}$ valence is from oxygen stoichiometry, the averaged $\mathrm{Ti}$ chemical valence should be the same for all samples, while SL2 has the lowest averaged Ti chemical valence compared to others. Hence, the dominant effect on the change in the $\mathrm{Ti}$ chemical valence should arise from the electronic charge transfer across the LSMO/STO interface. 

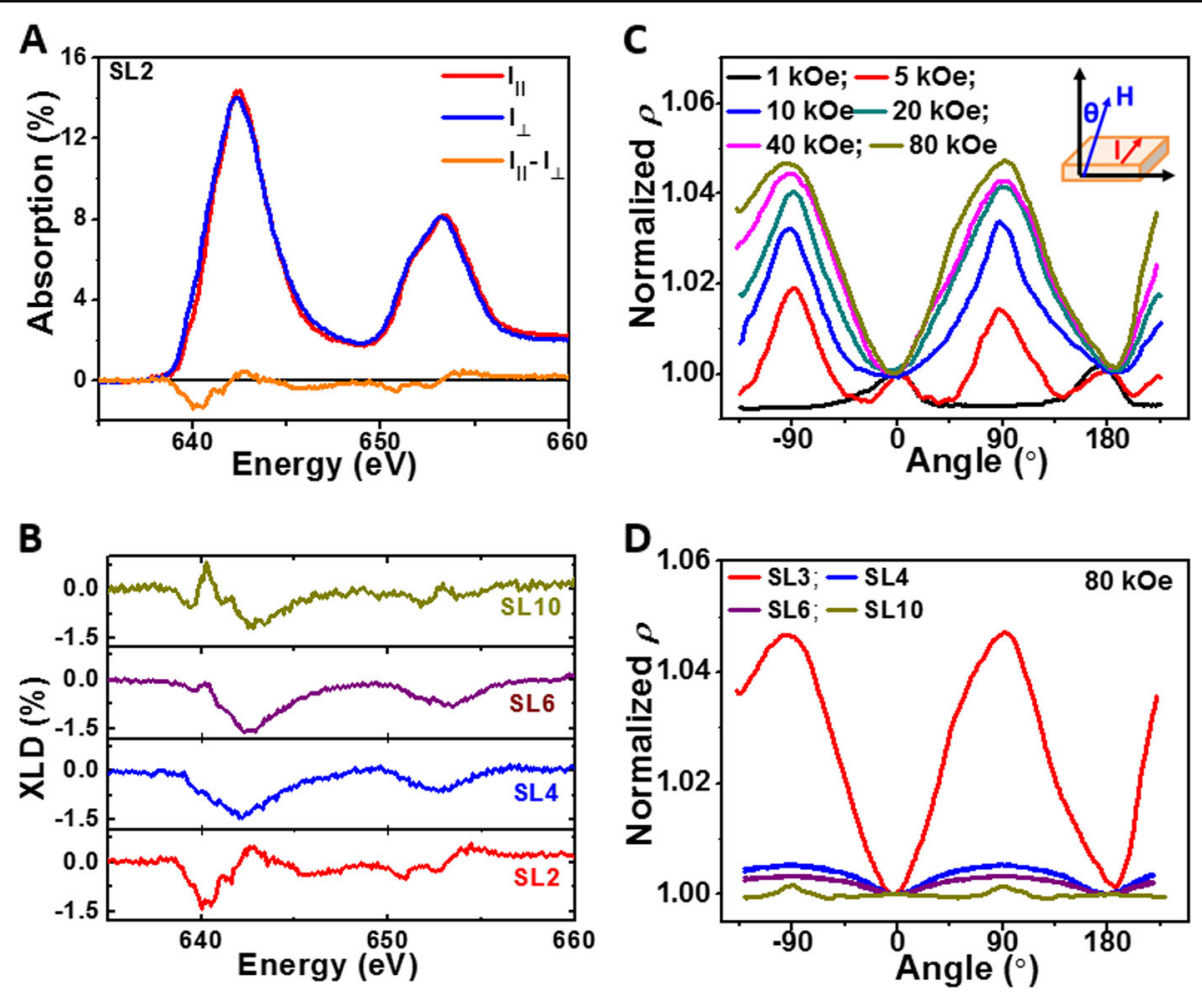

Fig. 4 Orbital occupancy and charge transfer in STO/LSMO superlattice films. a The detailed XAS curves for SL2, and the summarized Mn L edge XLD for four samples is shown in (b). The curves for different samples are vertically shifted for comparison. $\mathbf{c}$ The effect of the magnetic field on the angular dependence of magnetoresistance (AMR) of SL3 at $T_{\mathrm{Re}}=0.04(10 \mathrm{~K})$. All curves are normalized to the resistance at $\theta=0^{\circ}$. The

measurement configuration is illustrated as the inset of (c), and the current is along the (100) direction; $\mathbf{d}$ Summary of AMR at $10 \mathrm{~K}$ with magnetic field of $80 \mathrm{kOe}$ for different samples: $T_{\mathrm{Re}}=0.04$ for SL3, SL4 and SL6, and $T_{\operatorname{Re}}=0.03$ for SL10

In addition, $\mathrm{Mn} L$ edge X-ray linear dichroism (XLD) was performed to study the electronic configuration, as shown in Fig. 4a, b. The parallel XLD $\left(I_{||}\right)$was measured with the wave vector of incident X-ray parallel to the normal direction of the film plane, and the electric field of the X-ray is parallel to the film plane. The perpendicular XLD $(I)$ was measured with the wave vector of incident $\mathrm{X}$-ray $70^{\circ}$ to the normal direction, and the electric field of the X-ray is $70^{\circ}$ to the film plane. The XLD was calculated as the intensity difference $\left(I_{||}-I\right)$ between the spectra measured with in-plane $\left(E_{||}\right)$and out-of-plane $(E)$ polarizations. The XLD was measured at $300 \mathrm{~K}$ for $n=2,4,6$, and $350 \mathrm{~K}$ for $n=10$. The difference of two $\mathrm{X}$-ray absorption spectra shows the difference of occupancy in the two $e_{\mathrm{g}}$ orbitals. The gradual decrease in intensity of the feature at $\sim 640 \mathrm{eV}$ is due to the gradual increase in relative occupancy of $3 d_{3 z^{2}-r^{2}}$ orbitals with decreasing $n$, as shown in Fig. 4b; and SL2 shows the preferred occupancy of $3 d_{3 z^{2}-r^{2}}$ orbitals ${ }^{65,66}$. The resultant depletion of the $3 d_{3 z^{2}-r^{2}}$ orbitals weakens the ferromagnetic double-exchange interaction ${ }^{65}$ and lowers the paramagnetic-to-ferromagnetic phase transition temperature in SL2.
The charge transfer with corresponding orbital occupancy has a strong effect on angular-dependent magnetoresistance (AMR) at the low temperature range. During AMR measurement, the magnetic field rotates from the out-of-plane to the in-plane direction and remains perpendicular to the current (along [100] direction) as shown in the inset of Fig. 4c. Around these temperature ranges, all films show in-plane magnetic anisotropy (Fig. S2). Normally the resistance minimum should occur with the magnetic field in the film plane $\left(\theta=90^{\circ}\right)$. However, with increasing magnetic field from 1 to $80 \mathrm{kOe}$, the resistance minimum point SL3 changes from the in-plane to the outof-plane $\left(\theta=0^{\circ}\right)$ directions. This phenomenon is related to orbital reconstruction ${ }^{67}$ with charge transfer across the LSMO/STO interface, especially the $3 d_{3 z^{2}-r^{2}}$ orbital occupancy, and the AMR increases with decreasing temperature $^{67}$. When the magnetic field is in the film plane, the $3 d_{3 z^{2}-r^{2}}$ band flattens at the Fermi level due to the Mn spin-orbit coupling and results in a larger resistivity compared to the resistivity with the magnetic field along the out-of-plane direction due to the decreasing electronic velocity. Figure $4 \mathrm{~d}$ shows that the AMR effect is largest for SL3, and the AMR effect decreases with 
increasing $n$. SL10 has a smaller AMR at the reduced temperature $T_{\mathrm{Re}}=0.03$ compared to others at $T_{\mathrm{Re}}=0.04$, suggesting that SL10 has the lowest AMR effect at the same reduced temperature among these SLs. Although the resistance of SL2 exceeds the low temperature measurement capability as shown in Fig. S11, the trend of AMR indicates that the effect of orbital reconstruction at the LSMO/STO interface increases with decreasing $n$ (enhanced interfacial coupling). Additional experimental results show that AMR with the currents along both [100] and [110] directions shows the same trend as discussed above. The large difference between AMR in SL4 with currents along the [100] and [110] directions may be related to the anisotropy in the space of electron density ${ }^{68}$, or the local anisotropy of spin-orbital coupling ${ }^{69}$ with orbital reconstruction at the LSMO/STO interface. The exact origin of the dependence of AMR on the current direction warrants further future work. See S11 for more details.

The origin of the preferred occupancy of $3 d_{3 z^{2}-r^{2}}$ at the heterostructure interface was attributed to arguments such as the interfacial symmetry breaking and new crystal structure phases ${ }^{53,62}$. Based on the existence of the charge transfer and AMR, a mechanism due to orbital hybridization with charge transfer across the interface ${ }^{70}$ is proposed (Fig. 5a). From the perspective of molecular orbitals, the interfacial bonding lowers the energy of $3 d$ orbitals, favoring the occupancy of the $3 d_{3 z^{2}-r^{2}}$ orbital due

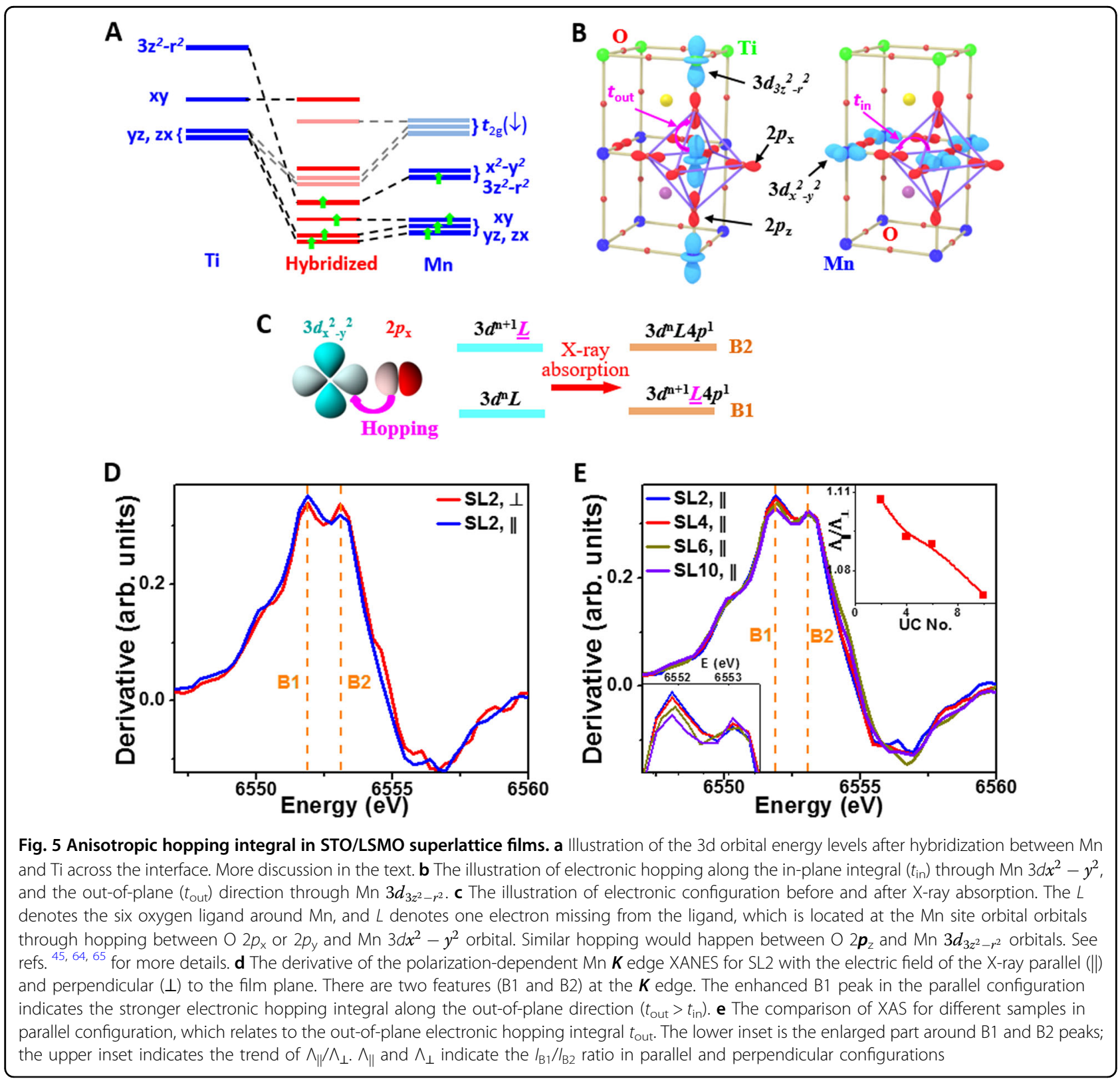


to a large spatial overlap. When this interfacial orbital forms, the electronegativity of interfacial bonds may facilitate the charge transfer between $\mathrm{Mn}$ and $\mathrm{Ti} 3 d$ orbitals. With the correlation between crystal structure and electronic structure in $3 d$ oxides, the preferred $3 d_{3 z^{2}-r^{2}}$ orbital occupancy may enlarge the out-of-plane $c_{\text {int }}$ to lower the total energy, as supported by the enlarged $c_{\text {int }} / a>1$ around the left LSMO/STO interface of each LSMO layer (Fig. 3). The oscillating pattern of $c_{\text {int }}$ for SL10 and SL6 (Fig. 3a, b) could be understood as follows. For one LSMO layer, the 1st UC LSMO at the left has a strong coupling with its left STO layer. Thus, the 1st UC LSMO has a large $c_{\text {int }}$ with charge transfer. The coupling decreases with increasing thickness of LSMO and $c_{\text {int }} / a$ decreases to $<1$. Along with the further increase in thickness with negligible charge transfer, $c_{\text {int }}$ continues to decrease due to in-plane tensile strain and reaches a minimum value at the right interface. At the right interface, the $c_{\text {int }} / a<1$ even with the existence of charge transfer. The subsequent 1st UC STO couples to the preceding LSMO layer with minimum $c_{\text {int }}$; with increasing thickness of STO, the $c_{\text {int }}$ increases until reaching the next STO/LSMO interface.

The above results suggest strong coupling around the LSMO/STO interface, and the existence of multiple LSMO/STO interfaces facilitates the charge transfer. With the increase in the interface number (decreasing $n$ ), the interfacial term $H_{\mathrm{i}}$ becomes more dominant. The trend of $H_{\mathrm{i}}$ could be addressed from the electronic hopping integral, as below. For a tight-binding Hamiltonian ${ }^{20}$ of the LSMO ultrathin film, $\sum_{R} t_{\alpha \beta}(R) e^{i K \cdot R}+\left(\frac{\lambda}{2}\right) \sigma(\theta, \phi)+$ $\xi L \cdot S$ was proposed to explain the magnetic anisotropy energy (MAE) according to density functional theory. The $t_{\alpha \beta}(R)$ represents the hopping integral from orbital $\alpha$ at the original site to orbital $\beta$ at site R. $K$ is the waver vector, $\lambda$ is the exchange splitting, the magnetization is along the $(\theta, \phi)$ direction, and $\sigma(\theta, \phi)$ is the vector of Pauli matrices times a unit vector in the direction $(\theta, \phi)$. $\xi$ represents the spin (S)-orbital $(L)$ coupling intensity. The structural change mainly affects $t_{\alpha \beta}(R)$, which in turn leads to the change in magnetic anisotropy. A high hopping integral is favored along the magnetic easy axis in a ferromagnetically ordered state. In the current work, the hopping integral along the in-plane $t_{\text {in }}$ and the out-of-plane $t_{\text {out }}$ direction is illustrated in Fig. 5b. The relative intensity of the hopping integral correlates with the detailed shape of the polarization-dependent Mn $K$ edge XANES ${ }^{51,71,72}$ as shown in Fig. 5c-e and Fig. S12. The electronic configuration of the $\mathrm{MnO}_{6}$ octahedron in the $\mathrm{B} 1$ state is proportional to the hopping integral, which could be used to characterize the in-plane and the out-of-plane hopping integral: the higher intensity ratio $\left(\Lambda_{\|}=\mathrm{I}_{\mathrm{B} 1} / \mathrm{I}_{\mathrm{B} 2}\right)$ in the parallel measurement with the polarization vector $(E$ vector of $\mathrm{X}$-ray) parallel to the film plane suggests a higher out-of-plane hopping integral $t_{\text {out }}$. For SL2, the out-ofplane hopping integral $t_{\text {out }}$ is higher than the in-plane hopping integral $t_{\text {in }}$ in Fig. $5 \mathrm{~d}$, induced by the enhanced $3 d_{3 z^{2}-r^{2}}$ orbital occupancy. With the increase in interface numbers (decreasing $n), \Lambda_{\|}$increases and the $\Lambda_{\|} / \Lambda_{\perp}$ ratio increases in Fig. 5e, indicating that the perpendicular contribution to the effective magnetic anisotropy increases with the increase in interface number (decreasing $n$ ), consistent with the trend of $H_{\mathrm{i}}$ with decreasing $n$, which causes $\sim 45^{\circ}$ rotation of effective magnetic anisotropy towards the out-of-plane direction from SL10 to SL2 at $T_{\mathrm{Re}}=0.87$. Please note that current work does not contradict ref. ${ }^{13}$, which proposed that the change in crystal structure- $\mathrm{MnO}_{6}$ rotation is the leading factor to affect orbital hybridization in a bilayer or trilayer system. Actually, current superlattice work also shows the change in crystal structure in terms of the lattice constant around the interface, and further study on electronic structure with charge transfer echoes with the change in lattice constant. The periodic thickness, number of interfaces and total thickness may play a role in the extent of the influence of the interfacial orbital hybridization on the out-of-plane magnetic anisotropy. These possible approaches to achieve a larger angular rotation in magnetic anisotropy warrant further investigation.

\section{Conclusions}

In summary, the variation in periodic thickness of the $\left(\mathrm{La}_{0.67} \mathrm{Sr}_{0.33} \mathrm{MnO}_{3}\right)_{n} /\left(\mathrm{SrTiO}_{3}\right)_{n}$ SLs affects the interfacial atomic arrangement and induced charge transfer and orbital hybridization. Increasing the number of interfaces enhances orbital hybridization, resulting in interfacial electronic charge transfer from Mn $3 d$ and Ti $3 d$ orbitals and corresponding rotation of the magnetic easy axis towards the out-of-plane direction for $n=2$ at $T_{\mathrm{Re}}=0.87$. This work demonstrates a promising approach of the use of SLs to control interface-induced properties of strongly correlated oxides in the development of novel magnetoelectronic devices.

\begin{abstract}
Acknowledgements
The research was supported by the Singapore Ministry of Education Academic Research Fund Tier 2 under the Project No. MOE2015-T2-1-016 and the Singapore National Research Foundation under CRP Award No. NRF-CRP102012-02. Work at Brookhaven National Laboratory was supported by the U.S. Department of Energy, Office of Basic Energy Science, Division of Materials Science and Engineering, under Contract No. DESC0012704. P.Y. is supported from SSLS via NUS Core Support C-380-003-003-001. Sector 20 facilities at the Advanced Photon Source and research at these facilities are supported by the U.S. Department of Energy - Basic Energy Sciences, the Canadian Light Source and its funding partners, the University of Washington, and the Advanced Photon Source. Use of the Advanced Photon Source, an Office of Science User Facility operated for the U.S. Department of Energy (DOE) Office of Science by the Argonne National Laboratory, was supported by the U.S. DOE under Contract No. DE-AC02-06CH1 1357.
\end{abstract}

\section{Author details}

'Department of Materials Science \& Engineering, National University of Singapore, 9 Engineering Drive 1, Singapore 117576, Singapore. ${ }^{2}$ Condensed Matter Physics \& Materials Science Division, Brookhaven National Laboratory, 
Upton, NY 11973, USA. ${ }^{3}$ Department of Physics and the Collaborative Innovation Center for Optoelectronic Semiconductors and Efficient Devices, Xiamen University, Xiamen 361005, China. ${ }^{4}$ Xiamen University Malaysia, 439000 Sepang, Selangor, Malaysia. ${ }^{5}$ Singapore Synchrotron Light Source (SSLS), National University of Singapore, 5 Research Link, Singapore 117603, Singapore. ${ }^{6}$ Advanced Photon Source, Argonne National Laboratory, Argonne, IL 60439, USA. ${ }^{7}$ NUSNNI-Nanocore, National University of Singapore, 2 Engineering Drive 3, Singapore 117581, Singapore. ${ }^{8}$ Department of Physics, National University of Singapore, 2 Science Drive 3, Singapore 117551, Singapore. ${ }^{9}$ Department of Electrical \& Computer Engineering, National University of Singapore, 4 Engineering Drive 3, Singapore 117583, Singapore. ${ }^{10}$ NUS Graduate School for Integrative Science and Engineering, National University of Singapore, 28 Medical Drive, Singapore 117456, Singapore

\section{Conflict of interest}

The authors declare that they have no conflict of interest.

\section{Publisher's note}

Springer Nature remains neutral with regard to jurisdictional claims in published maps and institutional affiliations.

Supplementary information is available for this paper at https://doi.org/ 10.1038/s41427-018-0084-8.

Received: 18 December 2017 Revised: 2 June 2018 Accepted: 26 June 2018. Published online: 25 September 2018

\section{References}

1. May, S. J. et al. Enhanced ordering temperatures in antiferromagnetic manganite superlattices. Nat. Mater. 8, 892-897 (2009).

2. Chakhalian, J. et al. Magnetism at the interface between ferromagnetic and superconducting oxides. Nat. Phys. 2, 244-248 (2006).

3. Chakhalian, J. et al. Orbital reconstruction and covalent bonding at an oxide. Interface Sci. 318, 1114-1117 (2007).

4. Ohtomo, A. \& Hwang, H. Y. A high-mobility electron gas at the $\mathrm{LaAlO}_{3} / \mathrm{SrTiO}_{3}$ heterointerface. Nature 427, 423-426 (2004).

5. Qin, Qi. Hang et al. Resistive switching in all-oxide ferroelectric tunnel junctions with ionic interfaces. Adv. Mater. 28, 6852-6859 (2016).

6. Grisolia, M. N. et al. Hybridization-controlled charge transfer and induced magnetism at correlated oxide interfaces. Nat. Phys. 12, 484 (2016).

7. Takamura, Y. et al. Competing interactions in ferromagnetic/antiferromagnetic perovskite superlattices. Phys. Rev. B 80, 180417(R) (2009).

8. Hoffman, J. et al. Oscillatory noncollinear magnetism induced by interfacial charge transfer in superlattices composed of metal oxides. Phys. Rev. X $\mathbf{6}$, 041038 (2016).

9. Garcia-Barriocanal, J. et al. Spin and orbital Ti magnetism at $\mathrm{LaMnO}_{3} / \mathrm{SrTiO}_{3}$ interfaces. Nat. Commun. 1, 82 (2010).

10. Bruno, F. Y. et al. Electronic and magnetic reconstructions in La0.7Sr0.3MnO3/ SrTiO3 heterostructures: a case of enhanced interlayer coupling controlled by the interface. Phys. Rev. Lett. 106, 147205 (2011).

11. Yang, F. et al. The effect of interfacial charge transfer on ferromagnetism in perovskite oxide superlattices. J. Appl. Phys. 111, 013911 (2012).

12. Rondinelli, J. M. \& Nicola, A. Spaldin, structure and properties of functional oxide thin films: insights from electronic-structure calculations. Adv. Mater. $\mathbf{2 3}$ 3363-3381 (2011).

13. Liao, Z. et al. Thickness dependent properties in oxide heterostructures driven by structurally induced metal-oxygen hybridization variations. Adv. Func. Mater. 27, 1606717 (2017).

14. He, J. et al. Control of octahedral tilts and magnetic properties of perovskite oxide heterostructures by substrate symmetry. Phys. Rev. Lett. 105, 227203 (2010).

15. Wang, Z. et al. Anomalously deep polarization in $\mathrm{SrTiO}_{3}(001)$ interfaced with an epitaxial ultrathin manganite film. Phys. Rev. B 94, 155307 (2016).

16. Aso, R. et al. Octahedral tilt propagation controlled by a-site cation size at perovskite oxide heterointerfaces. Cryst. Growth Des. 14, 2128-2132 (2014).
17. Liang, Q. et al. Dimensionality controlled octahedral symmetry-mismatch and functionalities in epitaxial $\mathrm{LaCoO}_{3} / \mathrm{SrTiO}_{3}$ heterostructures. Nano Lett. 15, 4677-4684 (2015)

18. Moon, E. J. et al. Effect of interfacial octahedral behavior in ultrathin manganite films. Nano Lett 14, 2509-2514 (2014).

19. Zhang, B. et al. Interfacial coupling-induced ferromagnetic insulator phase in manganite film. Nano Lett. 16, 4174-4180 (2016).

20. Liao, Z. et al. Controlled lateral anisotropy in correlated manganite heterostructures by interface-engineered oxygen octahedral coupling. Nat. Mater. 15, 425-431 (2016).

21. Daisuke, $\mathrm{K}$. et al. Tuning magnetic anisotropy by interfacially engineering the oxygen coordination environment in a transition metal oxide. Nat. Mater. 15 432-437 (2016)

22. Dagotto, E., Hotta, T. \& Moreo, A. Colossal magnetoresistant materials: the key role of phase separation. Phys. Rep. 344, 1-153 (2001).

23. Yang, $D$. et al. Pressure-induced magnetic transition in manganite $\left(\mathrm{La}_{075} \mathrm{Ca}_{025} \mathrm{MnO}_{3}\right)$. Phys. Rev. Lett. 102, 237201 (2009).

24. $\mathrm{Lin}, \mathrm{W}-\mathrm{N}$. et al. Electrostatic modulation of $\mathrm{LaAlO}_{3} / \mathrm{SrTiO}_{3}$ interface transport in an electric double-layer transistor. Adv. Mater. Interfaces 1, 1300001 (2014).

25. Zhang, $\mathrm{S}$. et al. Electric-field control of nonvolatile magnetization in $\mathrm{Co}_{40} \mathrm{Fe}_{40} \mathrm{~B}_{20} / \mathrm{Pb}\left(\mathrm{Mg}_{1 / 3} \mathrm{Nb}_{2 / 3}\right)_{0.7} \mathrm{Ti}_{0.3} \mathrm{O}_{3}$ structure at room temperature. Phys. Rev Lett. 108, 137203 (2012).

26. Ham, K-M. et al. Raman-active phonons in thin a- and c-axis-oriented $\left(\mathrm{YBa}_{2}-\right.$ $\left.\mathrm{Cu}_{3} \mathrm{O}_{7}\right) \mathrm{m}\left(\mathrm{PrBa}_{2} \mathrm{Cu}_{3} \mathrm{O}_{7}\right) \mathrm{n}$ superlattices. Phys. Rev. B 50, 16598 (1994).

27. Popović, Z., Satpathy, S. \& Martin, R. M. Origin of the two-dimensional electron gas carrier density at the $\mathrm{LaAlO}_{3}$ on $\mathrm{SrTiO}_{3}$ interface. Phys. Rev. Lett. 101, 256801 (2008).

28. Kim, M. S. et al. Structure, magnetic, and transport properties of Ti-substituted $\mathrm{La}_{0.7} \mathrm{Sr}_{0.3} \mathrm{MnO}_{3}$. Phys. Rev. B 71, 014433 (2005).

29. Vailionis, A. et al. Misfit strain accommodation in epitaxial $A B O 3$ perovskites: lattice rotations and lattice modulations. Phys. Rev. B 83, 064101 (2011).

30. Loetzsch, R. et al. The cubic to tetragonal phase transition in $\mathrm{SrTiO}_{3}$ single crystals near its surface under internal and external strains. Appl. Phys. Lett. 96 071901 (2010).

31. Lee, P. W. et al. Hidden lattice instabilities as origin of the conductive interface between insulating $\mathrm{LaAlO}_{3}$ and $\mathrm{SrTiO}_{3}$. Nat. Commu. 7, 12773 (2016).

32. Nakagawa, N., Hwang, H. Y. \& Muller, D. A. Why some interfaces cannot be sharp. Nat. Mater. 5, 204-209 (2006).

33. Salluzzo, M. et al. Origin of interface magnetism in $\mathrm{BiMnO}_{3} / \mathrm{SrTiO}_{3}$ and $\mathrm{LaAlO}_{3} /$ $\mathrm{SrTiO}_{3}$ heterostructures. Phys. Rev. Lett. 111, 087204 (2013).

34. Javier, G-B. et al. "Charge Leakage" at $\mathrm{LaMnO}_{3} / \mathrm{SrTiO}_{3}$ interfaces. Adv. Mater. 22 627-632 (2010)

35. Vaz, C. A. F., Walker, F. J., Ahn, C. H. \& Ismail-Beigi, S. Intrinsic interfacial phenomena in manganite heterostructures. J. Phys. Condens. Matter 27, 123001 (2015).

36. Seo, J. W. et al. Tunable magnetic interaction at the atomic scale in oxide heterostructures. Phys. Rev. Lett. 105, 167206 (2010).

37. Bunau, O. \& Joly, Y. Self-consistent aspects of $x$-ray absorption calculations. J. Phys. Condens. Matter 2, 345501 (2009).

38. Yves Joly et al. Electron population analysis by full-potential X-ray absorption simulations. Phys. Rev. Lett. 82, 2398 (1999).

39. Tsui, F. et al. Strain-dependent magnetic phase diagram of epitaxial $\mathrm{La}_{0.67} \mathrm{Sr}_{0.33} \mathrm{MnO}_{3}$ thin films. Appl. Phys. Lett. 76, 2421 (2000).

40. Johnsony, M. T. et al. Magnetic anisotropy in metallic multilayers. Rep. Prog. Phys. 59, 1409-1458 (1996).

41. Ikeda, S. et al. A perpendicular-anisotropy CoFeB-MgO magnetic tunnel junction. Nat. Mater. 9, 721-724 (2010).

42. Ding, J. et al. Manganite/cuprate superlattice as artificial reentrant spin glass, Adv. Mater. Interfaces 3, 1500676 (2016).

43. Nakajima, R. et al. Electron-yield saturation effects in L-edge $x$-ray magnetic circular dichroism spectra of Fe, Co, and Ni. Phys. Rev. B 59, 6421 (1999).

44. Lee, J.S. et al. Hidden magnetic configuration in epitaxial $\mathrm{La}_{1-x} \mathrm{Sr}_{x} \mathrm{MnO}_{3}$ films Phys. Rev. Lett. 105, 257204 (2010).

45. $\mathrm{Yu}$, P. et al. Interface ferromagnetism and orbital reconstruction in $\mathrm{BiFeO}_{3}$ $\mathrm{La}_{0.7} \mathrm{Sr}_{0.3} \mathrm{MnO}_{3}$ heterostructures. Phys. Rev. Lett. 105, 027201 (2010).

46. Park, J. et al. Quantifying interlayer exchange coupling via layer-resolved hysteresis loops in antiferromagnetically coupled manganite/nickelate superlattices. Appl. Phys. Lett. 95, 102504 (2009).

47. Huang, D. J. et al. Spin and orbital magnetic moments of $\mathrm{Fe}_{3} \mathrm{O}_{4}$. Phys. Rev. Lett. 93, 077204 (2004) 
48. Chen, $C$. et al. Experimental confirmation of the X-ray magnetic circular dichroism sum rules for iron and cobalt. Phys. Rev. Lett. 75, 152 (1995).

49. Di Yi et al. Tuning perpendicular magnetic anisotropy by oxygen octahedral rotations in $\left(\mathrm{La}_{1-x} \mathrm{Sr}_{x} \mathrm{MnO}_{3}\right) /\left(\mathrm{Srl}_{\mathrm{rO}}\right)$ superlattices. Phys. Rev. Lett. 119, 077201 (2017).

50. Glazer, A. M. The classification of tilted octahedra in perovskites. Acta Crystallogr., Sect. B: Struct. Crystallogr. Cryst. Chem. 28, 3384 (1972).

51. Zhang, B. et al. Strain modulated anisotropic electronic charge transfer in perovskite $\mathrm{Pr}_{0.67} \mathrm{Sr}_{0.33} \mathrm{MnO}_{3}$ thin films. Phys. Rev. B 89, 195140 (2014).

52. May, S. J. et al. Quantifying octahedral rotations in strained perovskite oxide films. Phys. Rev. B 82, 014110 (2010).

53. Di $\mathrm{Yi}_{\mathrm{i}}$ et al. Atomic-scale control of magnetic anisotropy via novel spin-orbit coupling effect in $\mathrm{La}_{2 / 3} \mathrm{Sr}_{1 / 3} \mathrm{MnO}_{3} / \mathrm{SrlrO}_{3}$ superlattices. PNAS 113, 6397-6402 (2016).

54. Hÿtch, M. J., Putaux, J-L. \& Pénisso, J-M. Measurement of the displacement field of dislocations to $0.03 \AA$ by electron microscopy. Nature 423, 270-273 (2003).

55. Lijun Wu et al. Nanostructures and defects in thermoelectric $\mathrm{AgPb}_{18} \mathrm{SbTe}_{20}$ single crystal. J. Appl. Phys. 105, 094317 (2009).

56. Hanghui, $C$. et al. Reversible modulation of orbital occupations via an interface-induced polar state in metallic manganites. Nano Lett. 14, 4965-4970 (2014).

57. Guo, H. et al. Interface-induced multiferroism by design in complex oxide superlattices, PNAS, E5062-E5069 (2017).

58. Adamo, C. et al. Effect of biaxial strain on the electrical and magnetic properties of (001) $\mathrm{La}_{0.7} \mathrm{Sr}_{0.3} \mathrm{MnO}_{3}$ thin films. Appl. Phys. Lett. 95, 112504 (2009).

59. Stoyanov, E., Langenhorst, F. \& Steinle-neumann, G. The effect of valence state and site geometry on $\mathrm{Ti}_{\mathrm{i}} \mathrm{L}_{2,2}$ and $\mathrm{O} \mathrm{K}$ electron energy-loss spectra of $\mathrm{Ti}_{x} \mathrm{O}_{y}$ phases. Am. Mineral. 92, 577-586 (2007).
60. Becerra-Toledo, A. E. et al. $\mathrm{c}(4 \times 2)$ and related structural units on the $\mathrm{SrTiO} 3$ (001) surface: scanning tunneling microscopy, density functional theory, and atomic structure. J. Chem. Phys. 136, 214701 (2012).

61. Gibert, M. et al. Interfacial control of magnetic properties at LaMnO3/LaNiO3 interfaces. Nano Lett. 15, 7355-7361 (2015).

62. Varela, M. et al. Atomic-resolution imaging of oxidation states in manganites. Phys. Rev. B 79, 085117 (2009).

63. Abbate, $\mathrm{M}$. et al. Controlled-valence properties of $\mathrm{La}_{1-x} \mathrm{Sr}_{x} \mathrm{FeO}_{3}$ and $\mathrm{La}_{1}$ ${ }_{x} \mathrm{Sr}_{x} \mathrm{MnO}_{3}$ studied by soft-x-ray absorption spectroscopy. Phys. Rev. B 46, 4511 (1992).

64. Wu, M. et al. Investigation of the multiplet structures and crystal field effects of $\mathrm{a} \mathrm{TiO}_{6} 3 d^{1}$ cluster based on configuration interaction calculations. J. Appl. Cryst. 50, 576-584 (2017).

65. Pesquera, D. et al. Surface symmetry-breaking and strain effects on orbital occupancy in transition metal perovskite epitaxial films. Nat. Commun. 3, 1189 (2012).

66. Tebano, A. et al. Evidence of orbital reconstruction at interfaces in ultrathin $\mathrm{La}_{0.67} \mathrm{Sr}_{0.33} \mathrm{MnO}_{3}$ films. Phys. Rev. Lett. 100, 137401 (2008).

67. Nemes, N. M. et al. Signatures of a two-dimensional ferromagnetic electron gas at the $\mathrm{La}_{0.7} \mathrm{Sr}_{0.3} \mathrm{MnO}_{3} / \mathrm{SrTiO}_{3}$ interface arising from orbital reconstruction Adv. Mater. 26, 7516 (2014).

68. Joshua, A. et al. Gate-tunable polarized phase of two-dimensional electrons at the $\mathrm{LaAlO}_{3} / \mathrm{SrTiO}_{3}$ interface. PNAS 110, 9633 (2013).

69. Dhesi, S. S. et al. Anisotropic spin-orbit coupling and magnetocrystalline anisotropy in vicinal Co films. Phys. Rev. Lett. 87, 067201 (2001).

70. Okamoto, S. Magnetic interaction at an interface between manganite and other transition metal oxides. Phys. Rev. B 82, 024427 (2010).

71. Tolentino, $\mathrm{H}$. et al. Anisotropy of the core-hole relaxation in $\mathrm{x}$-ray-absorption spectroscopy as probed in square planar cuprates. Phys. Rev. B 45, 8091 (1992).

72. Zhou, Y. et al. Strongly correlated perovskite fuel cells. Nature 534, 231-234 (2016). 